\title{
Die Wirklichkeit visueller Modelle
}

"Nur wenige Ausdrücke«, so konstatierte bereits Nelson Goodman, „werden im populären und wissenschaftlichen Diskurs undifferenzierter gebraucht als $>$ Modell. Ein Modell ist etwas, das man bewundert oder dem man nacheifert, ein Muster, ein passender Fall, ein Typ, ein Prototyp, ein Exemplar, ein Modell in Originalgröße, eine mathematische Beschreibung - nahezu alles von einer nackten Blondine bis zu einer quadratischen Gleichung. "1 Es hat den Anschein, als solle Nelson Goodman mit seiner kritischen Bemerkung Recht behalten. Denn tatsächlich ist der Umfang des Begriffs >Modell erstaunlich weit: Wir sprechen gleichermaßen vom Atommodell wie vom Aktmodell, vom Gesellschaftsmodell und vom Flugzeugmodell - doch meinen wir jeweils sehr unterschiedliche Dinge.

Gegen solche Unübersichtlichkeit hilft ein Blick auf die Herkunft der heutigen Begriffsverwendung. Der lateinische Ausdruck modulus, auf den unser Begriff ,Modell zurückgeht, ist die Verkleinerungsform von modus, dem Maß. Die Rede von einem modulus diente schon in der Antike nicht allein als Bezeichnung für die ideale Proportion eines Gebäudes; zugleich konnte mit dem modulus der Anspruch erhoben werden, ein umfassendes, ja sogar ein universales Maß zu bezeichnen. Die Symmetrien und Verhältnisse dieses Maßes sollten sowohl für den Kosmos im Großen wie auch im Kleinen, das heißt den Menschen, gelten. Eine praktische Verwendung fand dieses universale Maß daher nicht allein in der anschaulichen, verkleinerten Darstellung eines geplanten Bauvorhabens, sondern überall da, wo ein proportional verkleinerter oder vergrößerter Nachbau als Abbild oder Vorbild eines originalen Gegenstands dienen konnte.

Unsere heutigen so vielschichtigen Verwendungsweisen des Begriffs >Modell bewahren diese alte, aus antiker Zeit geerbte Vorstellung eines idealen Maßes. Wenn wir von Modellorganismen, von Rollenmodellen oder modellhaften Handlungen sprechen, so meinen wir hierbei idealisierte Prototypen oder mustergültige Beispiele, die nicht allein als ein Vorbild oder ein Abbild für etwas anderes dienen, sondern in denen sich vielmehr ein Maß widerspiegelt, das eine sehr viel weiter reichende, ja sogar eine allgemeine Geltung beanspruchen soll und kann. Die konkrete Form mathematischer oder naturwissenschaftlicher Modelle etwa ist einzig ein Mittel, um allgemeingültige Verhältnisse, Beziehungen oder Strukturen - kurz: um Gesetze greifbar und begreifbar zu machen, die für einen ganzen Anwendungsbereich als bindend gelten. Solche Modelle geben Regeln vor, an denen sich die Vielfalt der Einzelphänomene ausrichten, messen und nicht zuletzt definieren lässt.

1 Nelson Goodman: Sprachen der Kunst. Entwurf einer Symboltheorie, [Indianapolis 1976], übers. von Bernd Philippi, Frankfurt am Main 1995, S. 164. 
Doch reicht die begriffsgeschichtliche Wurzel unserer gegenwärtigen Redeweisen vom `Modell $\ll$ weiter. Bereits in der Antike trat der Begriff des `modulus` nicht allein in philosophischen Kontexten, also im Zusammenhang mit einer theoretischen Verständigung über die Welt auf. Denn auch für eine Vielzahl - das Wissen wie die Künste betreffender - Praktiken war der modulus ein unverzichtbarer Begriff. Hier waren Modelle stets in einen Verwendungskontext eingebettet. Und es besteht Grund zu der Annahme, dass diese Ausrichtung auf die kulturelle Praxis der eigentliche Grund für die Ausbildung von Modellen gewesen ist. Denn selbst wenn Modelle als ein Vorbild, ein Abbild oder eine Idealisierung dienen sollen, so bleiben sie doch stets dem Kontext der durch sie ermöglichten Praktiken verpflichtet. Modelle sind keine statischen Repräsentationen. Sie sind vielmehr Werkzeuge, die bestimmte Handlungen überhaupt erst ermöglichen. Es ist der Vollzug dieser Handlungen, der die eigentliche Rechtfertigung von Modellbildungen ausmacht. Weder theoretisch noch historisch interessierte Analysen von Modellen dürfen daher diese Verwurzelung in der Praxis aus den Augen verlieren.

Gefragt werden muss daher stets nach dem Zweck oder den verschiedenen Zwecken eines Modells. Die Antworten hierauf liegen zumeist in überraschend offener Weise auf der Hand: So lassen sich an einem Atommodell Theorien über den Aufbau der Materie entwickeln und zugleich in äußerst anschaulicher Weise Einsichten hierüber gewinnen. Modelle scheinen jedenfalls weit weniger komplex, als dies etwa für symbolische Repräsentationen - zum Beispiel für die einen Laien schnell überfordernde Formelsprache der Naturwissenschaften - der Fall ist. Selbst bei einem so anschaulichen Artefakt wie einem Gemälde wird, grob verallgemeinernd gesagt, der Schwerpunkt auf Aspekten der Darstellung und der Repräsentation liegen. Gegenüber seinen möglichen Verwendungsweisen verhält sich ein solches Bild dahingegen sehr häufig recht unbestimmt. Deutlicher noch: Das Gemälde kann sich von den Kontexten seines Gebrauchs radikal emanzipieren. Die unüberschaubare Vielzahl alter Sakralkunst, die schon lange ihre liturgischen Funktionen abgestreift hat und sich nun in den Kathedralen unserer säkularisierten Zeit, den Museen, befindet, führt dies eindrücklich vor Augen. Ein Modell hingegen kann sich nur schwerlich vom Zweck seiner Verwendung emanzipieren, ohne hierbei einen substantiellen Verlust zu erleiden.

Um zu verstehen, was Modelle sind und welche Rolle sie für uns spielen, muss mithin ein ganz besonderes Augenmerk auf ihren Verwendungszusammenhang gelegt werden. Betroffen sind hiervon zuallererst die epistemischen Funktionen des Modells, das heißt seine Bedeutung für die Prozesse der Wissensproduktion sowie der Kommunikation über komplexe Sachverhalte. Die konkrete Gestalt von Modellen erlaubt es uns, Strukturen zu begreifen, die zuvor unbestimmt und abstrakt waren. Erkenntnis ist hierbei jedoch nicht so sehr eine Sache der nachvollziehenden Beschreibung von Formen. Die durch das Modell vermittelte Form ist vielmehr der Ausgangspunkt unserer Annäherung an das gestellte Problem. Unser Zugriff auf das Objekt wird durch das Modell nicht allein vermittelt, sondern durch dieses überhaupt erst ermöglicht. Ein Modell von etwas zu besitzen heißt daher nicht, einzig bestimmte, bereits beobachtete Merkmale davon einzufangen. 
Mit Modellen verfügen wir über höchst wirksame Instrumente, mit denen wir Dinge manipulieren und Entwicklungen voraussagen wollen. Anhand von Modellen wird daher nicht allein etwas bereits Bekanntes beschrieben. Mit ihnen konstruieren wir vielmehr eine bestimmte Wirklichkeit: die Wirklichkeit des Modells.

Auf den Philosophen Gilbert Ryle geht die Unterscheidung von zwei Arten des Wissens zurück; eine Unterscheidung, die im Grunde jedoch schon sehr viel älter ist. Ryle spricht von einem "Knowing that « und einem »Knowing how «. ${ }^{2}$ Ins Deutsche übersetzt lässt sich diese Unterscheidung durch die Opposition von "Wissen « und "Können « kennzeichnen. Zu wissen, dass etwas der Fall ist, bedeutet, von einer bestimmten Aussage überzeugt zu sein und eine korrekte Beschreibung geben zu können. Wenn jemand weiß, dass ein bestimmter Raum ein Fenster hat, dann kann er eine Beschreibung von diesem Raum geben. Wenn jemand hingegen weiß, wie etwas funktioniert oder aber wie man etwas macht, so bedeutet das nicht unbedingt, dass er dies in einer Beschreibung zusammenfassen kann: Wenn jemand weiß, wie man einen Raum wohnlich einrichtet oder wie man einen Motor auseinander nimmt und wieder zusammenbaut, dann besitzt er eine Fähigkeit, ein Können, das sich vor allem an seinen Handlungen und deren Ergebnissen messen lassen wird.

Wissen und Erkenntnis, die durch Modelle vermittelt werden, entsprechen insbesondere dieser zweiten Kategorie des »Knowing how». Betrachten wir dazu das Beispiel der anschaulichen Lehrmodelle, die zur Erklärung von komplizierten Maschinen oder ganzen Prozessabläufen verwendet werden: Am Modell einer Dampfmaschine lernen wir nicht so sehr, dass bestimmte Aussagen auf sie zutreffen, sondern wir lernen, wie diese Maschine funktioniert und wie sie bedient oder auch manipuliert werden kann. Man erwirbt ein Handlungswissen, eine Fähigkeit, mit dem modellierten Gegenstand richtig umzugehen. Man lernt, Handlungen angemessen oder, wie Ryle es nennt, »intelligent « auszuführen. Die Tatsache, dass ein anschauliches Lehrmodell einzig ein idealisiertes Abbild der echten Maschine ist, bleibt hiervon unbenommen. Es ist ein Mittel für den eigentlichen Zweck des Modells, die Funktionsweise der Maschine und den Umgang mit ihr zu erlernen.

Ihren größten Wert für die Produktion eines »Knowing how« gewinnen Modelle jedoch aufgrund ihrer konkreten physischen Präsenz; und dies heißt vor allem: aufgrund ihrer visuellen Präsenz. Am anschaulichen Modell können Verfahrens- und Verwendungsweisen erprobt und manipuliert werden, die am Objekt selbst nicht möglich sind. In bestimmten Handlungen können Modelle die Rolle eines Ersatzobjektes einnehmen und so über den Umweg von Ersatzhandlungen ein Verständnis vom Objekt selbst vermitteln. So wird zum Beispiel das Architekturmodell verschoben, korrigiert und verworfen; Handlungen, die als Ersatz für das Verschieben, Korrigieren und Verwerfen des tatsächlichen Bauwerkes gelten müssen. Der praktische Umgang mit einem Modell des Bauwerks lässt uns verstehen,

2 Gilbert Ryle: The Concept of Mind, [London 1949], London 1990, S. 26-60. 
wie es, einmal gebaut, als ein architektonischer Körper wirken und wie es sich zwischen anderen Gebäuden eingliedern könnte. Das Architekturmodell ist daher alles andere als ein statisches Vorbild für ein zukünftiges Gebäude. Es verkörpert vielmehr ein Wissen über dessen Veränderbarkeit, hält Alternativen bereit und unterliegt einer fortlaufenden Transformation. ${ }^{3}$ Einem Modell, das demgegenüber dennoch als endgültig angesehen werden soll, gehen stets komplexe Prozesse des Modellierens voraus, in welchen eine Vielfalt von Möglichkeiten erprobt und ausgelotet worden ist.

Die Anziehungskraft visueller Modelle beruht jedoch nicht nur darauf, dass sie Gegenständen, die andernfalls im Unbestimmten bleiben müssten oder die nicht ohne weiteres verfügbar sind, eine handhabbare und anschauliche Form geben. Entscheidenden Wert gewinnen visuelle Modelle, da sie unüberschaubare $\mathrm{Zu}$ sammenhänge in eine endliche Menge von Elementen gliedern und so Komplexität reduzieren helfen. Anhand von Modellen versuchen wir nicht allein die Wirklichkeit zu gliedern, vielmehr beschreiben wir mit Modellen diese Wirklichkeit als eine aus einzelnen Teilen zusammengesetzte. Modelle sind daher nicht Beschreibungen oder Darstellungen von etwas, sondern sie stellen uns die Bausteine, mit deren Hilfe wir etwas beschreiben oder darstellen, zur Verfügung. Sie sind Werkzeuge für eine Beschreibung, ohne selbst eine Beschreibung sein zu müssen. Modelle haben häufig einen stark ontologischen Charakter. Indem sie uns vermitteln, wie wir mit einem Objekt umgehen können, und indem sie zugleich seine elementaren Teile bestimmen, legen sie fest, was ein Objekt ist, und nicht nur, welche Eigenschaften es hat. Sie definieren also nicht zuerst, welche Aussagen über ein Objekt richtig sind, sondern welche Aussagen überhaupt sinnvoll sind. Von den "Bahnen" eines Elektrons kann man erst sprechen, wenn das entsprechende Modell vorausgesetzt wird.

Die Logik eines Modells definiert die Bestimmung des Gegenstandes, von dem oder für den es ein Modell ist. Doch bedarf das Modell einer konkreten Form, also eines Mediums durch das es in Erscheinung tritt und durch das es vermittelt wird. Visuelle Modelle unterliegen hierbei notwendigerweise der Logik des Visuellen; sie sind abhängig von den Formen, den Traditionen und den Verwendungsweisen des gewählten Mediums. Und umgekehrt entfalten visuelle Modelle eine eigene, nur schwer zu bändigende visuelle Kraft. Ihre Anschaulichkeit besitzt ein Eigenleben: Visuelle Modelle legen Deutungen nahe, sie betonen und verdecken Unterschiede und schließen andere Deutungen aus. Die Interpretation und das Wissen, das wir durch Modelle gewinnen, beruht immer auch auf einer Interpretation der eigenen Wahrnehmung. Über visuelle Modelle nachzudenken heißt daher stets auch, die Möglichkeiten und Grenzen visueller Wahrnehmung in den Blick zu nehmen, die sich hierbei eröffnenden Optionen einer Logik des Visuellen zu bestimmen und diese auf visuelle Modelle zu erweitern. Aus Goodmans ein-

3 Am Beispiel von Sankt Peter in Rom hat Horst Bredekamp einen solchen Prozess fortlaufender Modellierung gezeigt. Horst Bredekamp: Sankt Peter in Rom und das Prinzip der produktiven Zerstörung. Bau und Abbau von Bramante bis Bernini, Berlin 2000. 
gangs zitierter kritischer Einschätzung lässt sich ableiten, dass ein Nachdenken über Modelle im Allgemeinen zunächst vor allem ein hohes Maß an Differenzierung notwenig macht. Die Reflexion über visuelle Modelle im Besonderen kann hiervon gewiss nicht ausgenommen werden. Der Gewinn, den ein solches Nachdenken über visuelle Modelle in Aussicht stellt, wird nicht zuletzt in einer näheren Akzentuierung des Sichtbaren zu suchen sein. Denn visuelle Modelle sind stets zugleich Modelle der Visualität. 
Ingeborg Reichle, Steffen Siegel, Achim Spelten (Hg.)

Visuelle Modelle

Wilhelm Fink 


\section{INHALT}

Ingeborg Reichle, Steffen Siegel, Achim Spelten

Die Wirklichkeit visueller Modelle ........................ 9

\section{Begriff und Metapher}

Bernd Mahr

Cargo.

Zum Verhältnis von Bild und Modell

Achim Spelten

Visuelle Aspekte von Modellen

Samuel Strehle

Evidenzkraft und Beherrschungsmacht.

Bildwissenschaftliche und soziologische Zugänge

zur Modellfunktion von Bildern $\ldots \ldots \ldots \ldots \ldots \ldots \ldots \ldots \ldots \ldots \ldots \ldots \ldots \ldots \ldots \ldots \ldots$

Tobias Schlechtriemen

Metaphern als Modelle.

Zur Organismus-Metaphorik in der Soziologie ................. 71

Philipp Ekardt

Benjamins Bekleidungsmodelle.

Strumpf und Rüsche als Topologien der Bildtheorie .............. 85

\section{EXPERIMENT UND Wissen}

ReINHARD WendLeR

Das Spiel mit Modellen.

Eine methodische Verwandtschaft künstlerischer Werk-

und molekularbiologischer Erkenntnisprozesse 
Sebastian Vincent Grevsmühl

Das modellierte Antlitz der Erde.

Zur Geschichte der Modellierungsstrategien

der Kontinentalverschiebung . .

Carolin Artz

Das Fotogramm als visuelles Modell?

Die Visualisierung nichtsichtbarer Strahlen

in wissenschaftlichen Fotografien um 1900

INGEBORg REICHLE

Lebendige Kunst oder Biologische Plastik?

Reiner Maria Matysiks Prototypenmodelle

postevolutionärer Organismen

FARbTAFELN

\section{MAß UND RAUM}

Catharina Manchanda

Modelle und Prototypen.

Ein Überblick ........................................... 179

Steffen Siegel

Modell-Räume.

Architektur, Photographie, Topoklasmus ...................... 197

Der Blick ins Modell

Ein Gespräch mit Damaris Odenbach ....................... 215

Katrin Käthe Wenzel

Brot und Bauten.

Drei künstlerische Experimente zu

verformbarer Architektur

Stefan Riekeles

Ikodynamische Kreuzfahrt.

Sichtbarkeit und Tarnung in einer Arbeit

von Knowbotic Research 


\section{Zeit und Struktur}

Annemieke R. Verboon

Einen alten Baum verpflanzt man nicht.

Die Metapher des Porphyrianischen

Baums im Mittelalter ................................. 251

Sebastian Gießmann

Graphen können alles.

Visuelle Modellierung und Netzwerktheorie vor 1900 .............. 269

Jens Gulden

Semantik in visuellen Modellen.

Räumliche Regularitäten und körperliche

Erfahrungsmuster als Bedeutungsträger visueller Modelle ............ 285

Inge Hinterwaldner

Simulationsmodelle.

Zur Verhältnisbestimmung von Modellierung und

Bildgebung in interaktiven Echtzeitsimulationen

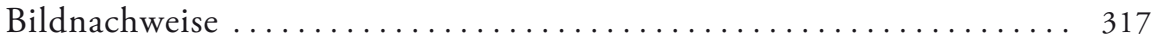

Autorinnen und Autoren ................................ 319 